\title{
Fast separations by capillary electrophoresis hyphenated to electrospray ionization time-of-flight mass spectrometry as a tool for arsenic speciation analysis
}

\author{
Claudia Niegel, ${ }^{a}$ Simon A. Pfeiffer, ${ }^{a}$ Marco Grundmann, ${ }^{a}$ Uriel Arroyo-Abad, ${ }^{b}$ Jürgen Mattusch ${ }^{b}$ \\ and Frank-Michael Matysik*a
}

Received 9th October 2011, Accepted 28th January 2012

DOI: 10.1039/c2an15944a

Fast capillary electrophoresis (CE) hyphenated to time-of-flight mass spectrometry (TOF-MS) of four organoarsenic species (glycerol oxoarsenosugar, sulfate oxoarsenosugar, arsenobetaine, arsenocholine) are presented using short length CE capillaries under high electric field strengths of up to $1.3 \mathrm{kV} \mathrm{cm}{ }^{-1}$ with small inner diameter (ID). The separation of arsenosugars by CE is demonstrated for the first time. An aqueous formic acid solution was employed as the background electrolyte (BGE) for the separation. Various acid concentrations were evaluated for their influence on migration times, separation efficiency as well as with regard to controlling the charge of the arsenic species. A $0.1 \mathrm{M}$ formic acid/ammonium formate buffer ( $\mathrm{pH}$ 2.8) proved to be suitable for the separation of the four species. A non-aqueous $\mathrm{BGE}$ was tested as an alternative buffer system for fast speciation analysis. Separation of arsenobetaine and arsenocholine could even be achieved within $10 \mathrm{~s}$ by pressure-assisted CE. Application of the optimized method for the analysis of extracts of a seagrass and a Wakame algae sample as well as the brown algae homogenate reference material IAEA-140/TM revealed a clear signal for the glycerol arsenosugar.

\section{Introduction}

Arsenic belongs to the elements most often subjected to speciation analysis ever since it has been generally accepted that its chemical, biological and toxicological properties, bioavailability and transport properties vary significantly with respect to its different oxidation states and chemical forms. Arsenic has always been associated with poisoning, and the inorganic forms, especially arsenate, are indeed highly toxic. However, toxicity is considered to decrease with the degree of methylation and eventually seems to become negligible. Thus, the determination of the total arsenic content gives no adequate information about the actual risk potential of a certain sample. Speciation analysis is an important tool to distinguish between the various species and evaluate potential impacts associated with the consumption of or exposure to the different species.

A great number and variety of different arsenic compounds have been identified in various environmental and biological matrices. Arsenosugars, arsenobetaine (AsB) and arsenoscholine $(\mathrm{AsC})$ are the predominant species in various kinds of seafood.

${ }^{a}$ University of Regensburg, Institute of Analytical Chemistry, Chemo- and Biosensors, University of Regensburg, 93053 Regensburg, Germany. E-mail: frank-michael.matysik@chemie.uni-regensburg.de; Fax: +49 941 943 4491; Tel: +499419434548

${ }^{b}$ UFZ - Helmholtz Centre for Environmental Research, Department of Analytical Chemistry, Permoserstrasse 15, 04318 Leipzig, Germany
While AsB has been found to be the main species in marine animals such as fish, molluscs and gastropods, arsenosugars primarily occur in (edible) marine macroalgae, especially in brown algae. AsC has been identified in various marine animals, e.g., shrimps, mussels and fish. It rarely contributes significantly to the total arsenic burden.

According to Feldmann and Krupp, arsenic-containing species can be generally divided into three groups: highly toxic, potentially non-toxic and (virtually) non-toxic species. ${ }^{1}$ The organoarsenicals $\mathrm{AsB}$ and $\mathrm{AsC}$ are believed to be non-toxic. However, it has been suggested that degradation of AsB results in an increase in the concentration of the more toxic tetramethylarsonium ion under certain conditions such as food processing. Possible negative health impacts of arsenosugar consumption are not known so far. They are generally considered to be non-toxic and regarded to be the end product of a detoxification process after intake of inorganic arsenic by marine organisms. However, their possible metabolites identified in human urine, e.g., dimethylarsinic acid (DMA), are suspected to exhibit a higher toxicity. ${ }^{2}$ It has also been suggested that arsenosugars can be further metabolized to AsC and AsB in marine sediments and marine animals, respectively. ${ }^{3}$ Extensive studies about the toxicity of arsenosugars and possible health implications are still due.

Arsenic speciation analysis is predominantly based on chromatographic separation techniques with ion-exchange 
chromatography (IC) generally being the method of choice. Separation protocols based on anion-exchange chromatography (AEC) and, less frequently, (strong) cation-exchange chromatography (CEC) have been established. Analysis by reversedphase high performance liquid chromatography (HPLC), frequently in combination with ion pairing, has also been reported. A number of reviews summarizing predominant methods for arsenic speciation analysis are available in the literature. ${ }^{4-6}$

The potential of capillary electrophoresis (CE) is often neglected when it comes to routine analysis. The main advantages include its low buffer consumption, high resolution power and low sample requirement. CE has been successfully employed for arsenic speciation analysis ${ }^{7-26}$ with its focus on elemental speciation analysis and the determination of inorganic species and the methylated arsenic acids (e.g., DMA, monomethylarsonic acid). AsB, AsC and phenylarsenic species (e.g., roxarsone) were frequently included in those studies. Common detectors employed for speciation analysis by $\mathrm{CE}$ include hydride generation inductively coupled plasma mass spectrometry (HGICP-MS), ICP atomic emission spectroscopy, UV, indirect UV and conductivity. CE offers the ability to separate anionic and cationic analytes within one run, thus providing ideal conditions for the simultaneous determination of a wide range of different arsenic species. By coupling it to molecular mass spectrometry, all species can be identified by switching from positive to negative mode in appropriate time segments.

So far, the determination of arsenosugars by CE has not been reported in the literature. Yeh and Jiang just briefly mention the possible detection of arsenosugars in extracts of a reference material oyster tissue by CE-ICP-MS in real world samples working in the counter-electroosmotic mode and under micellar electrokinetic chromatographic conditions. ${ }^{8}$ The predominately occurring oxoarsenosugars contain a dimethylarsinoyl group that is protonated at $\mathrm{pH}$ values below 4 and show the potential to be determined by $\mathrm{CE}$. AsB and AsC have been separated by $\mathrm{CE}$ before. ${ }^{15-26}$ However, the number of studies dealing with the analysis of $\mathrm{AsB}$ and $\mathrm{AsC}$ by $\mathrm{CE}$ hyphenated to MS detection is very limited. ${ }^{23-26}$ One of the main issues of CE-ESI-MS measurements is the generally long migration times since the setup generally requires the use of long capillaries (up to $100 \mathrm{~cm}$ or longer), especially when working with commercial instruments. Typical total separation times for AsB and AsC range between $16 \mathrm{~min}^{23}$ and $20 \mathrm{~min}^{24}$ Meermann et al. reported the separation of AsB within $3 \mathrm{~min}$ using a $50 \mathrm{~cm}$ capillary and a borate/ methanol background electrolyte (BGE). ${ }^{25}$ Schramel et al. employed a more elaborate separation protocol that was based on sample stacking. ${ }^{23}$ In addition, they investigated the effect of pressure-driven separation approaches (high and low pressure) on migration times, peak symmetry, resolution and detection limits. However, pressure was not applied until $10 \mathrm{~min}$ after injection. The separations reported in the literature so far still show potential for optimization.

In a recent paper, fast and efficient electrophoretic separations using short capillaries with small IDs coupled to time-of-flight MS (TOF-MS) were reported accomplishing separations of various catecholamines within $30 \mathrm{s.}^{27} \mathrm{~A}$ homologous series of six hyaluronan oligosaccharides could be separated within $65 \mathrm{~s}$ under counter-electroosmotic conditions. ${ }^{28}$ The general potential of the application of short capillaries for $\mathrm{CE}$ has recently been reviewed. ${ }^{29}$ General advantages and disadvantages of short capillaries have been discussed in detail. ${ }^{27}$ The fast acquisition rates of TOF-MS provide high peak resolution. Moreover, the high mass accuracy of TOF-MS offers an additional verification tool, especially since there is a lack of (commercially) available arsenosugar standards. Thus, the hyphenation of CE to TOFMS represents a powerful and complementary alternative to IC and HPLC analysis.

In this study we present the potential of aqueous and nonaqueous CE-ESI-TOF-MS for fast arsenic speciation analysis using short fused silica capillaries and applying high electrical field strength. The separation of arsenosugars by capillary zone electrophoresis is demonstrated for the first time. A pressuremobilized separation approach is briefly illustrated as a method to realize separations of cationic arsenic species within $10 \mathrm{~s}$. Finally, the optimized method is successfully applied to the analysis of various algae samples.

\section{Experimental}

\section{Instrumentation}

All CE experiments were carried out using a laboratory-made CE instrument, consisting of a high voltage power supply (model HCN 7E-35000, F.u.G. Elektronik, Rosenheim-Langenpfunzen, Germany), a control unit, a Plexiglas safety box and a manual sample injection system. The CE system was coupled to a micrOTOF-MS (Bruker Daltonics, Bremen, Germany). The separation capillary was inserted into a coaxial sheath liquid sprayer (Agilent Technologies, Waldbronn, Germany).

\section{Chemicals and materials}

Fused silica capillaries ( 25 and $50 \mu \mathrm{m}$ i.d., $360 \mu \mathrm{m}$ outer diameter) were purchased from Polymicro Technologies (Phoenix, AZ, USA). All reagents used were of analytical grade or higher. Deionised water supplied by an Astacus system (MembraPure, Bodenheim, Germany) was used throughout this study. HPLCMS grade isopropanol and ammonium acetate were from Carl Roth (Karlsruhe, Germany). Methanol (HPLC gradient grade), acetonitrile (HPLC gradient grade), formic acid, acetic acid and ammonium hydroxide were purchased from Merck (Darmstadt, Germany). Arsenobetaine and arsenocholine were obtained from Wako Chemicals GmbH (Neuss, Germany). The glycerol oxoarsenosugar and the sulfate oxoarsenosugar were acquired from K. A. Francesconi (Karl-Franzens University, Graz, Austria). Caffeine was an Avocado Research Chemicals Ltd product purchased from ABCR GmbH \& Co. KG (Karlsruhe, Germany). The sea plant homogenate reference material IAEA-140/ TM was kindly donated by J. R. Oh (International Atomic Energy Association, Monaco). The powdered seagrass was taken from an interlaboratory study. ${ }^{30}$ The Wakame algae (Wakame Taipan) were bought at a local Asian store (importer: Arrow Trading Hamburg) and pulverized (particle size $>30 \mu \mathrm{m}$ ).

\section{CE-ESI-TOF-MS analysis}

For separations in aqueous buffers $25 \mu \mathrm{m}$ ID capillaries (27 or $29.5 \mathrm{~cm}$ ) were used. Pressure-assisted separations and 
separations in non-aqueous buffers were performed in $50 \mu \mathrm{m}$ ID capillaries $(28.5 \mathrm{~cm})$. The sheath liquid consisted of a mixture of isopropanol/water $(50: 50, \mathrm{v} / \mathrm{v})$ acidified with formic acid $(0.2 \%)$. The sheath liquid flow rate was set to $8 \mu \mathrm{L} \mathrm{min}^{-1}$. The TOF-MS source parameters were optimized for the enhanced detection of the arsenosugars. Source parameters were adjusted if studies were limited to the determination of $\mathrm{AsB}$ and $\mathrm{AsC}$. The nebulizer gas pressure was set to 1.0 and 1.4 bar. The dry gas flow was $4.0 \mathrm{~L}$ $\mathrm{min}^{-1}$ and the dry gas temperature was $190^{\circ} \mathrm{C}$. Separations were conducted using either aqueous or a non-aqueous BGE. Aqueous BGEs were prepared of concentrated formic acid by appropriate dilution. If necessary, $\mathrm{pH}$ was adjusted using $25 \%$ ammonium hydroxide, thus giving ammonium formate/formic acid buffers. The non-aqueous CE (NACE) buffer contained $1 \mathrm{M}$ acetic acid and $10 \mathrm{mM}$ ammonium acetate in acetonitrile. Manual injection was accomplished as described by Grundmann and Matysik. ${ }^{28}$ The hydrodynamic injection procedure consisted of the manual transfer of the capillary from the buffer vial to the sample vial for 3 or $10 \mathrm{~s}$, and back. A separation voltage of $35 \mathrm{kV}$ was applied. Caffeine was used as EOF marker.

\section{Pressure-assisted separations}

Pressure-assisted separations were carried out in $50 \mu \mathrm{m}$ ID capillaries $(28.5 \mathrm{~cm})$. After manual injection for $3 \mathrm{~s}$, the capillary was moved back to the $4 \mathrm{~mL}$ buffer vial containing $4 \mathrm{~mL}$ of BGE and the cap was screwed tightly before the separation voltage was turned on. After $5 \mathrm{~s}$, pressure (approx. 1 bar) was applied by injecting air into the vial from outside the Plexiglas CE box using a syringe and tubing.

\section{Extraction of real samples}

A simple extraction protocol was used for the extraction of a dried Wakame and seagrass powder as well as a brown algae homogenate reference material (IAEA-140/TM, Fucus sp., Sea Plant Homogenate) which is no longer on the market. $100 \mathrm{mg}$ of algae sample were weighed into $15 \mathrm{~mL}$ PP centrifuge tubes. $5 \mathrm{~mL}$ of deionised water were added and the sample was thoroughly mixed by vortexing for a few minutes. Afterwards the samples were extracted by ultrasonication at $30{ }^{\circ} \mathrm{C}$ for $3 \mathrm{~h}$. After centrifugation for $30 \mathrm{~min}$ at $10000 \mathrm{rpm}$, the supernatant was filtered through a membrane syringe filter (regenerated cellulose, $0.45 \mu \mathrm{m}$, phenomenex, Aschaffenburg, Germany) and the filtrate was transferred to $1.5 \mathrm{~mL}$ Eppendorf tubes. $300 \mu \mathrm{L}$ of the filtrate were transferred to $0.5 \mathrm{~mL}$ Eppendorf tubes and evaporated to dryness in a slight nitrogen stream. The residue was re-dissolved in $29.2 \mu \mathrm{L}$ deionised water. $0.8 \mu \mathrm{L}$ of a $10^{-2} \mathrm{M}$ caffeine standard was added to the extracts before analysis. Unless samples were analyzed the same day, they were stored frozen.

\section{Results and discussion}

\section{General separation aspects}

A mixture containing the four arsenic species AsB, AsC, AsS-OH and $\mathrm{AsS}^{-\mathrm{SO}_{4}}$ (Fig. 1) and caffeine as EOF marker was analyzed using short capillaries with small IDs $(25 \mu \mathrm{m}$ and $50 \mu \mathrm{m})$. Conventional underivatized carbohydrates are commonly separated under basic $\mathrm{CE}$ conditions (e.g., sodium hydroxide,
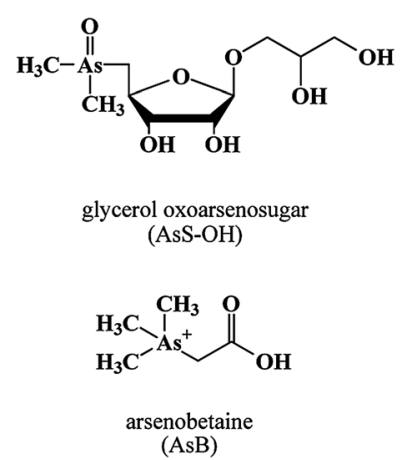

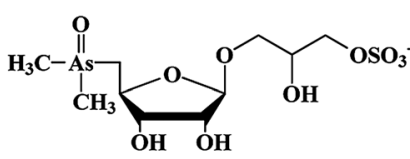

sulfate oxoarsenosugar $\left(\mathrm{AsS}-\mathrm{SO}_{4}\right)$

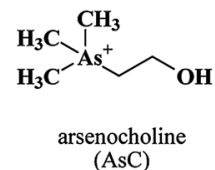

Fig. 1 Structures of the four organoarsenic species analyzed.

triethylamine) or in the presence of borate, to form complexes, thus, often depending on separation protocols that are not suitable for hyphenation with mass spectrometric detection. We evaluated the suitability of a simple buffer system that has been successfully employed before for fast separations. ${ }^{27}$ Aqueous buffers are most commonly used for CE separations. However, non-aqueous and mixed aqueous-organic solvent buffers show great potential, especially with respect to selectivity and sensitivity. The application of non-aqueous buffers has been intensively studied in the past.

\section{Fast separation of arsenic species in aqueous buffers}

The effect of buffer concentration and composition on the migration times of a mixture containing the four arsenic species $\mathrm{AsB}, \mathrm{AsC}, \mathrm{AsS}-\mathrm{OH}$ and $\mathrm{AsS}-\mathrm{SO}_{4}$ and caffeine as EOF marker

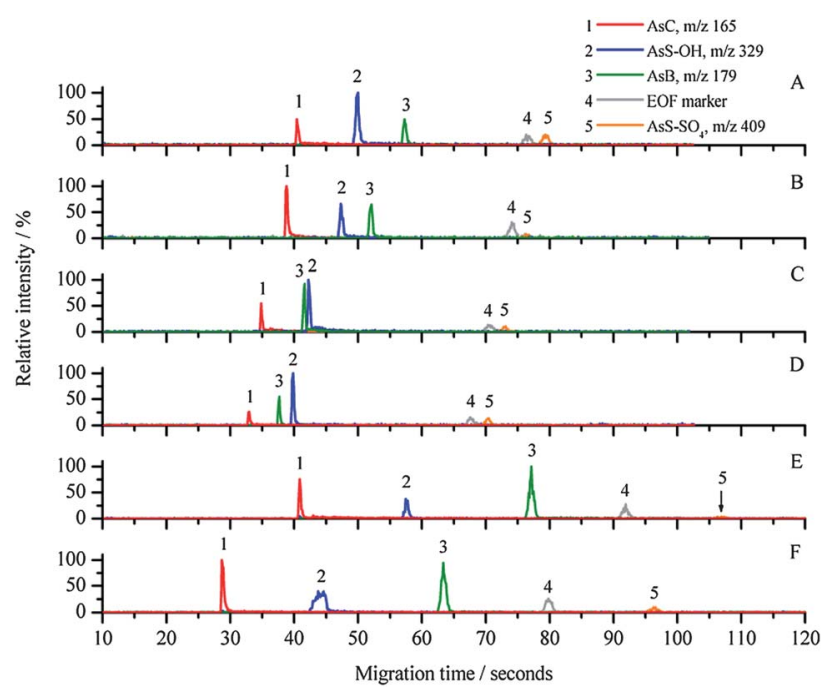

Fig. 2 Effect of the BGE concentration and composition on the migration times of the four organoarsenic species AsC (6 mg L $\left.{ }^{-1} \mathrm{As}\right), \mathrm{AsB}(6 \mathrm{mg}$ $\left.\mathrm{L}^{-1} \mathrm{As}\right)$, AsS-OH (3 mg L $\left.{ }^{-1} \mathrm{As}\right)$ and AsS-SO 4 (6.5 mg L $\left.{ }^{-1} \mathrm{As}\right)$. Extracted ion traces are shown. The buffer used was aqueous formic acid of $0.05 \mathrm{M}$ (A), $0.1 \mathrm{M}$ (B), $0.5 \mathrm{M}$ (C), $1 \mathrm{M}$ (D), $0.05 \mathrm{M}$ adjusted to $\mathrm{pH} 3$ (E), and $0.1 \mathrm{M}$ adjusted to $\mathrm{pH} 3(\mathrm{~F})$. Caffeine $(80 \mu \mathrm{M})$ was used as EOF marker. Separations were carried out at $35 \mathrm{kV}$ in a $29.5 \mathrm{~cm} \times 25 \mu \mathrm{m}$ capillary. The sheath liquid flow rate was $8 \mu \mathrm{L} \mathrm{min}{ }^{-1}$ and the nebulizer gas pressure was 1.4 bar. Detection and separation parameters were kept constant for all measurements. Samples were injected hydrodynamically for $10 \mathrm{~s}$. 
was studied under aqueous acidic conditions using a formic acid system as BGE (Fig. 2). To optimize the separation and allow for baseline separation of the different species as well as the separation from the EOF marker, different formic acid concentrations ranging from $0.05 \mathrm{M}$ to $2.5 \mathrm{M}$ were investigated. At formic acid concentrations of $1 \mathrm{M}$ and above, currents increased significantly $(>50 \mu \mathrm{A})$ requiring either the lowering of the separation voltage or the use of smaller i.d. capillaries.

The ionization state of AsB $\left(\mathrm{p} K_{\mathrm{a}} 2.18^{31}\right)$ depends on $\mathrm{pH}$. With decreasing $\mathrm{pH}$, the concentration of the zwitterionic species is reduced, thus AsB is present as the positively charged species. The $\mathrm{p} K_{\mathrm{a}}$ value of the dimethylarsinoyl moiety has been experimentally determined to be $3.85 .^{32,33}$ Therefore, the positive net charge of AsS-OH under the separation conditions applied is due to the protonation of the dimethylarsinoyl group. The overall acid-base property of a molecule is primarily determined by the functional groups in the aglycone. ${ }^{34}$ The sulfuric acid ester moiety in the side chain of $\mathrm{AsS}_{-} \mathrm{SO}_{4}$ shows a strong acidic property. Thus, at $\mathrm{pH} 3$ this arsenosugar is separated from the neutral EOF marker. Instead of being completely converted to its

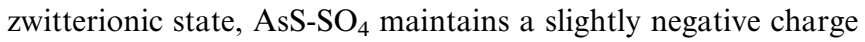
during the $\mathrm{CE}$ separation. Increasing the formic acid concentration to $2.5 \mathrm{M} \mathrm{HCOOH}$ (results not shown) did not further enhance the protonation of AsS-SO ${ }_{4}$ to give a positively charged species during the $\mathrm{CE}$ separation. Therefore, it can be concluded that the protonation of the negatively charged functional group in the aglycone of $\mathrm{AsS}^{-\mathrm{SO}_{4}}$ occurs during the electrospray ionization process.

As can be seen in Fig. 2, migration times decrease with increasing buffer concentration. For AsS-OH the relative migration remained constant while AsB migrates faster and eventually its peak is shifted before that of AsS-OH. Moreover, higher BGE concentrations result in narrower peaks but the positively charged species migrate closer together and there is a greater gap between positively charged and neutral analytes. As expected, peak heights increased considerably for AsS-OH and AsB at higher formic acid concentrations. For BGE concentrations of $2.5 \mathrm{M}$ the separation voltage was reduced to $25 \mathrm{kV}$ (results not shown). Compared to the separation in $1 \mathrm{M}$ formic acid buffer at $25 \mathrm{kV}$, the positively charged species were well separated from each other. However, those conditions result in comparatively long total analysis times (140 s).

The increase of the BGE concentrations requires the use of capillaries with smaller ID $(<50 \mu \mathrm{M})$ to prevent distortions or even the collapse of separations resulting from excessive Joule heating. However, problems that arise when reducing the ID to very small values include low loading capacity, high flow-induced backpressure and an increased risk of clogging. Sample injection for capillaries with IDs below $15 \mu \mathrm{m}$ requires the application of additional pressure ${ }^{27}$ or an electrokinetic approach. To keep the separation system simple, and since higher BGE concentrations did not enhance the total protonation of $\mathrm{AsS}_{-} \mathrm{SO}_{4}$, formic acid concentrations above $0.5 \mathrm{M}$ were not considered further.

The influence of the buffer $\mathrm{pH}$ was only evaluated for $0.05 \mathrm{M}$ and $0.1 \mathrm{M}$ formic acid, respectively. The BGE $\mathrm{pH}$ was adjusted to a value of 3.0 with $25 \%$ ammonium hydroxide to give formic acid/ammonium formate-based BGEs. As can be seen in Fig. 2 all four analytes and the EOF marker are almost evenly separated from each other. This is advantageous with regard to the analysis of real samples. However, peak shapes deteriorate at lower buffer concentrations with the positively charged species showing some peak tailing.

In a different approach, inorganic modifiers, i.e., inorganic salts such as ammonium or lithium chloride, were added to the BGE. However, this did not improve the separation and detec-

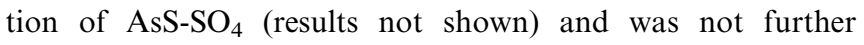
evaluated.

For $0.1 \mathrm{M}$ formic acid adjusted to $\mathrm{pH} 3$, the peak shape of AsS-OH was not satisfying. By decreasing the buffer $\mathrm{pH}$ to 2.8 , a significant improvement of the peak shapes was observed. Thus, $0.1 \mathrm{M} \mathrm{HCOOH}$ at $\mathrm{pH} 2.8$ was used as the final BGE. Shortening the capillary by $2.5 \mathrm{~cm}$ to a total length of $27 \mathrm{~cm}$ resulted in the separation of the four arsenic species and the EOF marker in less than 1 minute (Fig. 3). This rather drastic increase in separation speed might be due to a further increase of the hydrodynamic flow associated with the suction effect of the CEMS interface. However, handling of the shorter capillary was less easy and a frequent instability of the total ion current (TIC) was observed. Thus, a minimum capillary length of $28 \mathrm{~cm}$ is recommended.

Repeatability of migration times of consecutive injections was between 0.4 and $0.6 \%$ (29.5 cm capillary, $n=8)$ and 1.3 and $2 \%$ (27 cm capillary, $n=9$ ), respectively for the individual species. Limits of detection (LODs) were determined to be 3 times the signal-to-noise ratio $(\mathrm{S} / \mathrm{N})$. LODs were $160 \mu \mathrm{g} \mathrm{L}^{-1}$ As for AsC, $180 \mu \mathrm{g} \mathrm{L}^{-1}$ As for AsC, $125 \mu \mathrm{g} \mathrm{L}{ }^{-1}$ As for AsS-OH and $790 \mu \mathrm{g} \mathrm{L}^{-1}$ As for AsS-SO ${ }_{4}$. Relative standard deviations (RSDs) of peak heights for consecutive injections (AsS-OH, $3 \mathrm{mg} \mathrm{L}^{-1}$ As; AsS$\left.\mathrm{SO}_{4}, 6.5 \mathrm{mg} \mathrm{L}^{-1} \mathrm{As}\right)$ ranged between 13 and $17 \%(29.5 \mathrm{~cm}$ capillary, $n=8$ ) and 14 and 29\% (27 cm capillary, $n=9$ ).

Common problems and errors associated with quantification in CE-MS are summarized by Ohnesorge et al. ${ }^{34}$ Briefly,

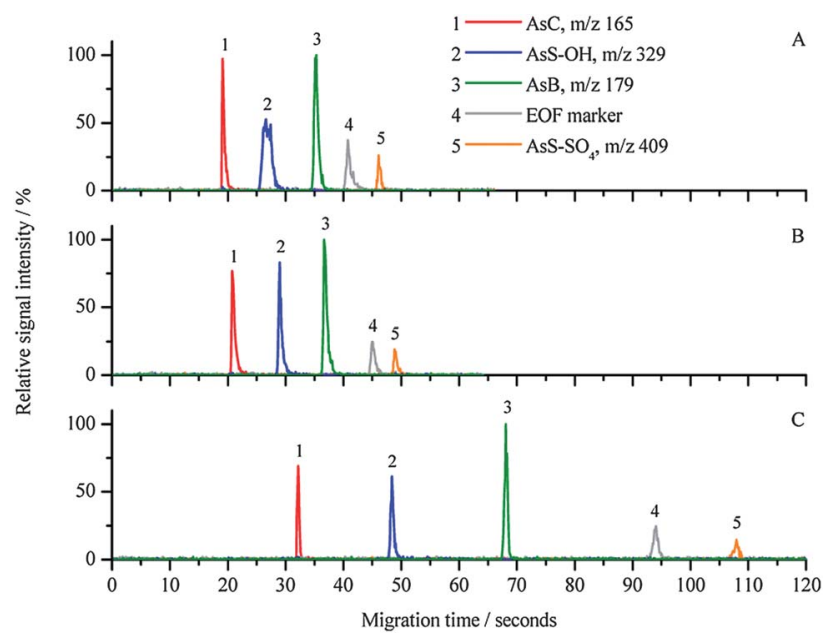

Fig. 3 Separations of four organoarsenicals in capillaries of two different lengths and at two different $\mathrm{pH}$ values. Extracted ion traces of

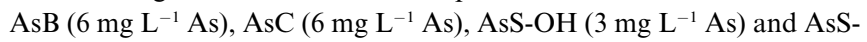
$\mathrm{SO}_{4}\left(6.5 \mathrm{mg} \mathrm{L}^{-1} \mathrm{As}\right)$ and caffeine $(80 \mu \mathrm{M}$, EOF marker) are shown. Separations were carried out at $35 \mathrm{kV}$ in a $25 \mu \mathrm{m} \times 27 \mathrm{~cm}$ in $0.1 \mathrm{M}$ $\mathrm{HCOOH}$ at $\mathrm{pH} 3$ (A) and $\mathrm{pH} 2.8$ (B) and a $25 \mu \mathrm{m} \times 29.5 \mathrm{~cm}$ capillary in $0.1 \mathrm{M} \mathrm{HCOOH}, \mathrm{pH} 2.8(\mathrm{C})$, respectively. Samples were injected for $10 \mathrm{~s}$ following the manual hydrodynamic injection protocol. ESI-TOF-MS conditions were the same as described before. 
quantification by external calibration is less reliable due to possible variations in ionization efficiency of the ESI source and possible ion suppression caused by matrix components. To considerably improve quantification, it is recommended to use isotopically labeled internal standards. However, these standards are not (commercially) available so far.

\section{Non-aqueous buffer system}

Non-aqueous electrophoresis (NACE) buffers offer a powerful alternative to enhance the selectivity and speed of CE separations. Moreover, they are completely compatible with MS detection. An established NACE buffer was initially evaluated for AsB and AsC only (Fig. 4).

Migration times increased significantly when changing from $0.05 \mathrm{M}$ formic acid adjusted to $\mathrm{pH} 2.8$ to the non-aqueous BGE. Furthermore, injections were more prone to interferences resulting in the frequent collapse of the separation current due to the lower surface tension of acetonitrile. The success rate of the injections was greatly influenced by the suction effect of the ESI sprayer which, in turn, mainly depends on the nebulizer gas pressure. By reducing the nebulizer gas pressure, injection failures were reduced. As can be seen in Fig. 4, intensity increased considerably for both arsenic species.

The non-aqueous buffer system was then evaluated for the analysis of the standard mix containing all four organoarsenicals (results not shown). This time, a decrease in migration times and a considerable increase in peak widths were observed. Whereas the glycerol sugar was detected, the sulfate analogue was not. However, the peak shape of AsS-OH was not satisfying, thus requiring further optimization.

The fundamental suitability and the potential of the NACE buffer for arsenic speciation analysis become obvious from Fig. 4. In general, the ionization behavior changes when using

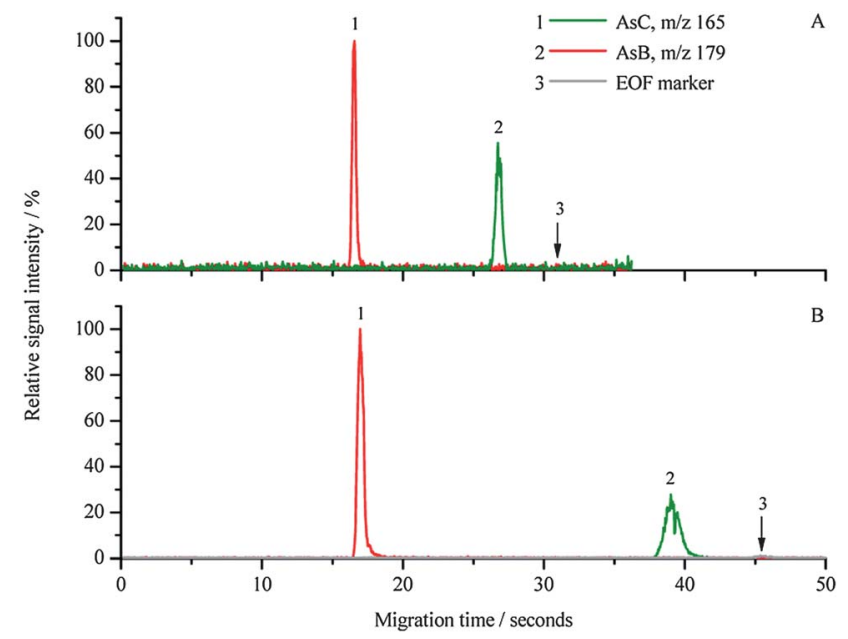

Fig. 4 Comparison of fast CE-MS measurements in aqueous (A) and non-aqueous (B) buffer systems. Extracted ion traces of $0.75 \mathrm{mg} \mathrm{L}^{-1} \mathrm{As}$ AsC and $0.75 \mathrm{mg} \mathrm{L}^{-1}$ As AsB are illustrated. $100 \mu \mathrm{M}$ caffeine was used as EOF marker. The sheath liquid flow was set to $8 \mu \mathrm{L} \mathrm{min}{ }^{-1}$ and the nebulizer gas pressure was 0.6 bar (NACE) or 1.0 bar (aqueous buffer). Samples were injected hydrodynamically for $3 \mathrm{~s}$. A separation voltage of $35 \mathrm{kV}$ was applied. Separations were carried out in a $50 \mu \mathrm{m} \times 28.5 \mathrm{~cm}$ capillary. a non-aqueous BGE. However, AsC should be least affected due to its permanent positive charge.

Non-aqueous solvents are frequently employed for sample preparation. Under aqueous separation conditions, these samples often require a buffer exchange. Thus, working with a NACE buffer is especially of interest with regard to improvement toward less elaborate sample preparation.

\section{Pressure-assisted separations}

To further speed up the separation, an additional pressure-driven separation approach was evaluated for the determination of AsB and $\mathrm{AsC}$ in aqueous and non-aqueous BGEs. This was accomplished by injecting air into the CE buffer vial via a syringe. The study revealed that migration times of the two species could be reduced to a total analysis time of less than $10 \mathrm{~s}$ for the NACE system and $14 \mathrm{~s}$ for the aqueous BGE while maintaining baseline separation (Fig. 5).

For the pressure-assisted separations, two approaches were tested, i.e., the application of pressure before or while the separation voltage was applied. The latter approach is illustrated in Fig. 5. Relative standard deviations of migration times for the NACE buffer were less than $1.9 \%$ for AsC and $6.0 \%$ for $\mathrm{AsB}(n=$ 6).

Excellent separation efficiencies were achieved for pressureassisted separations with peak widths at a half maximum height of $0.28 \mathrm{~s}(\mathrm{AsC})$ and $0.42 \mathrm{~s}(\mathrm{AsB})$ for the NACE buffer and $0.65 \mathrm{~s}$ $(\mathrm{AsC})$ and $0.66 \mathrm{~s}(\mathrm{AsB})$ for separations in aqueous BGE. The lowest achievable separation time is limited by the peak widths. To further reduce peak widths, capillaries with smaller i.d.s were tested (results not shown). However, for smaller i.d. capillaries, higher pressures were required that could not reproducibly be applied. Thus, there is a limit at which the gain in time does not compensate for the necessary conditions and expenditure to

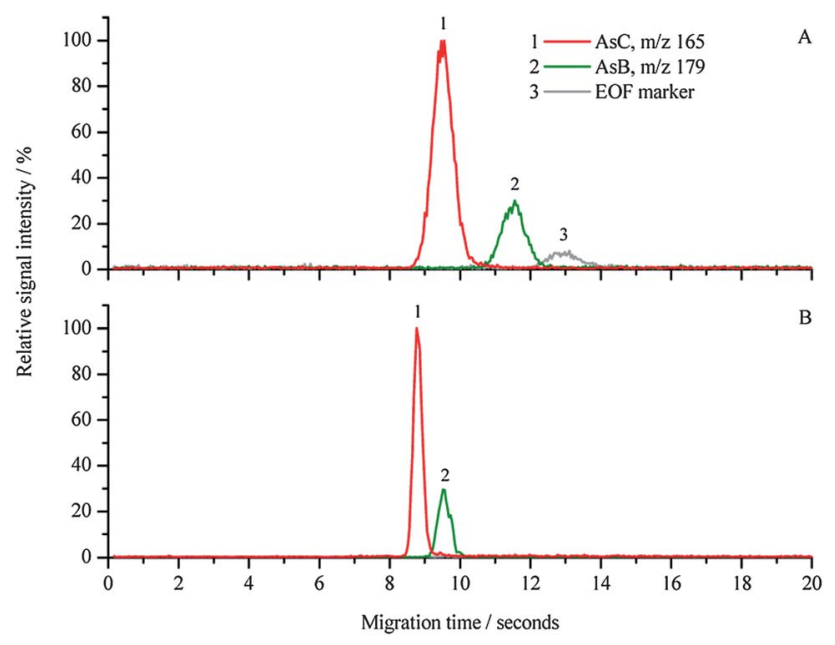

Fig. 5 Very fast separations by pressure-assisted CE-ESI-TOF-MS in an aqueous (A) and a non-aqueous (B) BGE. Extracted ion traces of 0.75 $\mathrm{mg} \mathrm{L}^{-1}$ As AsC and $0.75 \mathrm{mg} \mathrm{L}^{-1}$ As AsB are illustrated. $100 \mu \mathrm{M}$ caffeine was used as EOF marker. Injection, separation and detection conditions were as noted in Fig. $4.4 \mathrm{~mL}$ of air were applied to the buffer vial containing $4 \mathrm{~mL}$ of buffer $5 \mathrm{~s}$ after the separation voltage of $35 \mathrm{kV}$ was applied. A $50 \mu \mathrm{m} \times 28.5 \mathrm{~cm}$ capillary was used. 


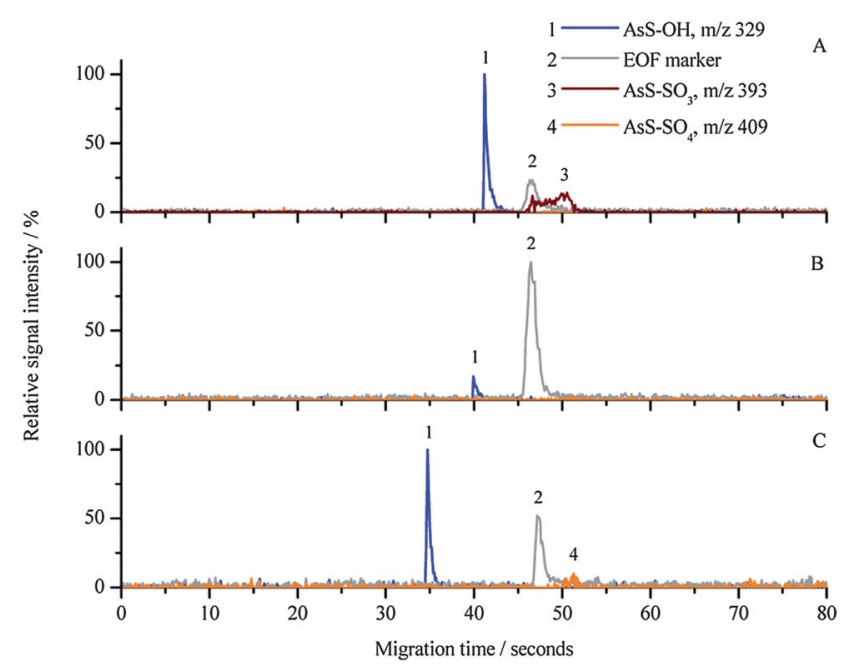

Fig. 6 Screening of real samples by fast CE-ESI-TOF-MS. Extracted ion traces of seagrass (A), Wakame (B) and brown algae reference material IAEA-140/TM (C) extracts in a $25 \mu \mathrm{m} \times 27 \mathrm{~cm}$ capillary under optimized separation conditions (as in Fig. 1) in $0.1 \mathrm{M} \mathrm{HCOOH}$ (pH 2.8) as BGE. For preconcentration, the extracts were evaporated to dryness and re-dissolved in water with a preconcentration factor of 10 . The sample was introduced into the capillary by hydrodynamic injection for $10 \mathrm{~s}$.

apply the appropriate pressure. Moreover, at very high pressures, a loss of resolution is observed since the flat flow profile is superimposed by a pressure-related parabolic one.

The pressure-assisted separation protocol and the nonaqueous buffer system were not applied to the analysis of real samples due to the more complicated handling.

\section{Real samples}

Marine algae, especially brown algae and particularly those of the Fucus genus, have been found to frequently contain high concentrations of arsenosugars. ${ }^{35}$ So far, there is no reference material available that has been certified for its arsenosugar content. However, the four most common oxoarsenosugars, i.e., AsS-OH, AsS-SO ${ }_{4}$, the sulfonate $\left(\mathrm{AsS}_{-} \mathrm{SO}_{3}\right)$ and the phosphate $\left(\mathrm{AsS}_{-} \mathrm{PO}_{4}\right)$ species, have been identified and quantified in the reference material IAEA-140/TM (Fucus sp.). ${ }^{36-39}$ In addition, in preliminary tests by AEC-TOF-MS we confirmed the presence of at least three of the most important arsenic-containing carbohydrate species (results not shown) in all algae samples considered during this study.

CE-MS analysis of the extracts under optimized separation conditions ( $0.1 \mathrm{M} \mathrm{HCOOH}, \mathrm{pH} 2.8)$ revealed a significant peak for the AsS-OH in all three extracts (Fig. 6). A very small peak for $\mathrm{AsS}_{-} \mathrm{SO}_{4}$ was detected in the IAEA-140/TM extract. Traces of the $\mathrm{AsS}_{-} \mathrm{SO}_{3}$ were identified in the seagrass sample. The AsS$\mathrm{PO}_{4}$ was not found in any of the extracts because of its overall low concentration. Relative distributions of the various arsenosugars in the individual algae samples differed from our results obtained during the preliminary tests as well as from values given in the literature. This is primarily due to the overall lower sensitivity of the species migrating after the EOF, i.e., the species containing a negatively charged functional group in the aglycone. In addition, degradation of $\mathrm{AsS}_{-} \mathrm{SO}_{4}, \mathrm{AsS}_{-}-\mathrm{SO}_{3}$ and AsS-
$\mathrm{PO}_{4}$ to AsS-OH might occur either during separation or, more likely, in the ESI source.

In the presence of matrix compounds, the migration of AsS$\mathrm{OH}$ was shifted towards the EOF marker and, thus towards longer times while the migration of the EOF marker and AsS$\mathrm{SO}_{4}$ remained constant. The presence of the arsenosugars was verified by standard addition and by isotope pattern matching. Sigma-FIT ${ }^{\mathrm{TM}}$, an additional software tool provided by the manufacturer, was applied to ensure the presence of the analytes. An elaborate clean-up procedure would be necessary to remove matrix compounds that influence the analytes' migration.

\section{Conclusions}

In this study, fast separations by $\mathrm{CE}-\mathrm{MS}$ of the four organic arsenic-containing species, the two arsenosugars (AsS-OH and AsS- $\mathrm{SO}_{4}$ ), AsB and $\mathrm{AsC}$, were successfully accomplished in short capillaries with IDs of $25 \mu \mathrm{m}$ and $50 \mu \mathrm{m}$ under high electric field strengths. Separations were performed in less than $2 \mathrm{~min}$ and were further reduced by decreasing the capillary length or by pressure application. Employing a pressure-assisted $\mathrm{CE}$ protocol, separations of $\mathrm{AsB}$ and $\mathrm{AsC}$ were achieved within $10 \mathrm{~s}$.

Arsenosugar analysis by CE-MS was performed for the first time. The separation protocol presented in this study offers a complementary technique due to the different selectivities of $\mathrm{CE}$ compared to (ion)chromatographic techniques. In addition, for injection sample volumes in the $\mathrm{nL}$ range are sufficient.

The suitability of aqueous and non-aqueous BGEs was evaluated. A simple aqueous BGE made of $0.1 \mathrm{M}$ formic acid $\mathrm{pH}-$ adjusted with ammonium hydroxide to 2.8 proved to be appropriate for the separation of the four organoarsenicals. Due to the more challenging injection in the case of NACE, the preferred protocol was based on the use of aqueous buffers.

The optimized method was successfully applied to the analysis of three algae samples, i.e., seagrass, Wakame, and the brown algae reference material IAEA-140/TM. AsS-OH was found to be the main arsenosugar species in all of them. Due to the overall lower sensitivity of AsS-SO $\mathrm{S}_{4}$, only traces could be identified in the reference material. In the seagrass extract, traces of $\mathrm{AsS}^{-\mathrm{SO}_{3}}$ were detected.

Further optimization of the developed method to additionally include inorganic and other relevant organic arsenic species would offer a powerful and fast screening approach for initial toxicity evaluations of real samples before extensive analysis. By hyphenation with TOF-MS, the presence of the analytes is verified without the need for (commercial) standards due to the instrument's high mass accuracy.

\section{Acknowledgements}

Financial support by the Deutsche Forschungsgemeinschaft (MA 1491/9-1) is gratefully acknowledged.

\section{Notes and references}

1 J. Feldmann and E. M. Krupp, Anal. Bioanal. Chem., 2011, 399, $1735-1741$.

2 C. Wei, W. Li, C. Zhang, M. Van Hulle, R. Cornelis and X. Zhang, J. Agric. Food Chem., 2003, 51, 5176-5182.

3 K. A. Francesconi, R. V. Stick and J. S. Edmonds, Experientia, 1990, 96, 464-466. 
4 C. Niegel and F.-M. Matysik, Anal. Chim. Acta, 2010, 657, 83-99.

5 Z. Gong, X. Lu, M. Ma, C. Watt and X. C. Le, Talanta, 2002, 58, 77-96.

6 K. A. Francesconi and D. Kuehnelt, Analyst, 2004, 129, 373-395.

7 B. Sun, M. Macka and P. R. Haddad, Electrophoresis, 2002, 23, 24302438.

8 D. R. Richardson, S. S. Kannamkumarath, R. G. Wuilloud and J. A. Caruso, Anal. Chem., 2004, 76, 7137-7142.

9 X.-B. Yin, X.-P. Yan, Y. Jiang and X.-W. He, Anal. Chem., 2002, 74, $3720-3725$.

10 J. F. López-Sánchez, M. D. Amran, M. D. Lakkis, F. Lagarde, G. Rauret and M. J. F. Leroy, Fresenius' J. Anal. Chem., 1994, 348, 810-814.

11 Y.-M. Huang and C.-W. Whang, Electrophoresis, 1998, 19, 21402144.

12 E. P. Gil, P. Ostapczuk and H. Emons, Anal. Chim. Acta, 1999, 389, 9-19.

13 M. L. Magnuson, J. T. Creed and C. A. Brockhoff, J. Anal. At. Spectrom., 1997, 12, 689-695.

14 K. Kutschera, A.-C. Schmidt, S. Köhler and M. Otto, Electrophoresis, 2007, 28, 3466-3476.

15 K. Van den Broeck and C. Vandecasteele, Microchim. Acta, 1998, 128, 79-85.

16 C. A. Suárez, G. C. L. Araújo, M. F. Giné, M. H. Kakazu and J. E. S. Sarkis, Spectrosc. Lett., 2009, 42, 376-382.

17 C.-F. Yeh and S.-J. Jiang, Electrophoresis, 2005, 26, 1615-1621.

18 G. Koellensperger, J. Nurmi, S. Hann, G. Stingeder, W. J. Fitz and W. W. Wenzel, J. Anal. At. Spectrom., 2002, 17, 1042-1047.

19 M.-W. Hsieh, C.-L. Liu, J.-H. Chen and S.-J. Jiang, Electrophoresis, 2010, 31, 2272-2278.

20 B. Michalke and P. Schramel, Electrophoresis, 1998, 19, 2220-2225.

21 J. A. Day, K. L. Sutton, R. S. Soman and J. A. Caruso, Analyst, 2000, 125, 819-823.

22 M. Van Holderbeke, Y. Zhao, F. Vanhaecke, L. Moens, R. Dams and P. Sandra, J. Anal. At. Spectrom., 1999, 14, 229-234.
23 O. Schramel, B. Michalke and A. Kettrup, J. Anal. At. Spectrom., 1999, 14, 1339-1342.

24 F. Kitagawa, K. Shiomi and K. Otsuka, Electrophoresis, 2006, 27, 2233-2239.

25 B. Meermann, M. Bartel, A. Scheffer, S. Trümpler and U. Karst, Electrophoresis, 2008, 29, 2731-2737.

26 L. Debusschere, C. Demesmay and J. L. Rocca, Chromatographia, 2000, 51, 262-268.

27 M. Grundmann and F.-M. Matysik, Anal. Bioanal. Chem., 2011, 400, 269-278.

28 M. Grundmann, M. Rothenhöfer, G. Bernhardt, A. Buschauer and F.-M. Matysik, Anal. Bioanal. Chem., 2012, 402, 26172623.

29 F.-M. Matysik, Anal. Bioanal. Chem., 2010, 397, 961-965.

$30 \mathrm{P}$. Fecher and C. Schuffenhauer, Poster Presentation, GDCh Jahrestagung Chemie 2003, München, 2003.

31 S. H. Hansen, E. H. Larsen, G. Pritzl and C. Cornett, J. Anal. At. Spectrom., 1992, 7, 629-634.

32 Y. Shibata, M. Morita and J. S. Edmonds, Agric. Biol. Chem., 1987, 51, 391-398.

33 G. Raber, K. A. Francesconi, K. J. Irgolic and W. Goessler, Fresenius' J. Anal. Chem., 2000, 367, 181-188.

34 J. Ohnesorge, C. Sänger-van de Griend and H. Wätzig, Electrophoresis, 2005, 26, 2360-2375.

35 J. T. van Elteren, Z. Šlejkovec, M. Kahn and W. Goessler, Anal. Chim. Acta, 2007, 585, 24-31.

36 A. D. Madsen, W. Goessler, S. N. Pedersen and K. A. Francesconi, J. Anal. At. Spectrom., 2000, 15, 657-662.

37 H. Castlehouse, C. Smith, A. Raab, C. Deacon, A. A. Meharg and J. Feldmann, Environ. Sci. Technol., 2003, 37, 951-957.

38 M. J. Ellwood and W. A. Maher, Anal. Chim. Acta, 2003, 477, 279291.

39 Z. Šlejkovec, A. R. Byrne, T. Stijve, W. Goessler and K. J. Irgolic, Appl. Organomet. Chem., 1997, 11, 673-682. 